\title{
Damage evolution in Saffil alumina short-fibre reinforced aluminium during tensile testing
}

\author{
R. Tavangar ${ }^{\mathrm{a}}$, L. Weber ${ }^{\mathrm{b}, *}$, A. Mortensen ${ }^{\mathrm{b}}$ \\ a Materials Research Laboratory, Sharif University of Technology, Teheran, Iran \\ ${ }^{\mathrm{b}}$ Laboratory for Mechanical Metallurgy, Swiss Federal Institute of Technology, CH-1015 Lausanne, Switzerland
}

Received 16 July 2004; received in revised form 29 November 2004; accepted 9 December 2004

\begin{abstract}
The evolution of microstructural damage during tensile deformation of pure aluminium reinforced with 10 vol. $\%$ alumina short fibres is studied by monitoring the evolution of density and Young's modulus as a function of tensile strain. It is found that Young's modulus drops rapidly until a strain $\varepsilon_{\mathrm{c}} \approx 3 \%$. The composite density remains virtually unchanged in this strain range. At strains above $\varepsilon_{\mathrm{c}}$, Young's modulus decreases more slowly while the density begins to decrease linearly, indicating void growth in the composite. It is shown that the drop in Young's modulus is linked to fragmentation of fibres aligned along the stress axis, while the decrease in density is related to void opening across fibre cracks.
\end{abstract}

(C) 2004 Elsevier B.V. All rights reserved.

Keywords: Aluminium matrix composites; Saffil short fibres; Damage evolution

\section{Introduction}

Metal matrix composites based on planar randomly oriented Saffil alumina short-fibre preforms have been used in automotive applications for more than 20 years [1,2]. The mechanical behaviour of this class of materials has, therefore, been the subject of several investigations, focused mainly on their tensile behaviour at ambient [3-13] and elevated temperatures [5,14,15], and their behaviour in creep [16-19], fatigue [20-24] and their fracture toughness [25-27].

In these, as in many other metal matrix composites, tensile deformation is, to a large extent, governed by the build-up of internal damage. The phenomenon is generally studied using two experimental approaches. The first is direct, consisting in qualitative microscopic description of the damage, e.g. observations of fibre breakage $[3,15]$ and void formation in the matrix [28]. In other investigations, indirect but quantitative measures of damage are reported, based on the measurement of variations with strain in certain composite physical

\footnotetext{
* Corresponding author. Tel.: +412169329 34; fax: +41216934664.

E-mail address: ludger.weber@epfl.ch (L. Weber).
}

properties, such as their density [28] or Young's modulus $[20,29]$.

The present work aims to contribute to our understanding of damage accumulation in these composites using both approaches simultaneously. Model composites of pure aluminium reinforced with $10 \%$ Saffil $^{\mathrm{TM}}$ short alumina fibres are strained in tension, and damage is characterised directly in terms of length distributions of broken fibre segments, and indirectly in terms of the evolution of Young's modulus and density of the composites. Relations between these different measures are then sought, with a goal to contribute a somewhat more complete understanding of how this class of composites accumulate internal damage as they deform. It is shown that there are, in these composites, transitions in damage accumulation mode with increasing strain.

\section{Experimental procedures}

\subsection{Material production}

The composites were produced by infiltrating preforms containing 10 vol.\% planar random oriented $\delta$-alumina fi- 


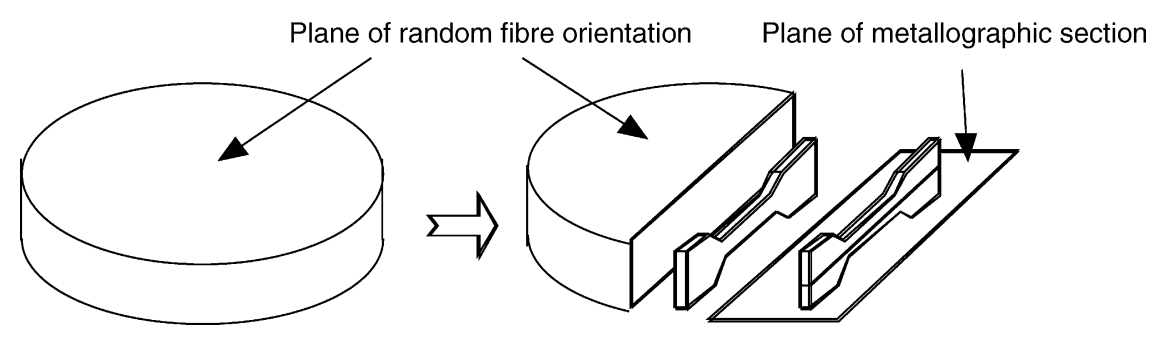

Fig. 1. Schematic of the as-infiltrated preform and the orientation of the samples as well as microscopic inspection for fibre segment length characterisation.

bres (SAFFIL RF grade, ICI Plc.) of average diameter $3 \mu \mathrm{m}$ with pure aluminium. Preform cohesion was ensured by addition of $3-4 \%$ silica binder. The preform was preheated to $300^{\circ} \mathrm{C}$ and infiltrated with commercially pure aluminium (99.8\% pure Al produced by IRALCO, Arak, Iran), superheated to $950^{\circ} \mathrm{C}$ using a pressure of $35 \mathrm{MPa}$, applied for $3 \mathrm{~min}$. No change in the preform thickness was observed along the pressing direction after infiltration; hence, the preform fibre volume fraction was retained in the composites.

\subsection{Tensile testing}

Flat dog-bone tensile specimens with gauge dimensions of $25 \mathrm{~mm} \times 6 \mathrm{~mm} \times 2 \mathrm{~mm}$ were extracted perpendicularly from the middle of the reinforced portion of the infiltrated castings as well as from the unreinforced matrix part of the squeeze cast ingot. The tensile axis of the composite specimen was oriented within the plane of random fibre orientation (Fig. 1). The surfaces of the specimens were polished down to 1000 grit emery paper in order to remove defects created during the cutting process.

Tensile tests were run on a Zwick $10 \mathrm{kN}$ screw-driven machine following ASTM standard B557M-84 save for the fact that the dog-bone samples were sub-sized. Longitudinal displacements were measured with a clip-on extensometer over a 10-mm gauge length. A nominal strain rate of $10^{-4} \mathrm{~s}^{-1}$ was used for all tests. The initial Young's modulus of the specimens was measured by repeated unloading-reloading cycles conducted between 30 and $70 \%$ of the load before unloading, after a small increment in deformation, typically $0.05 \%$ total strain [30].

\subsection{Metallography}

Optical microscopy was used to examine the general structure and fibre distribution in the composites. In order to investigate the mechanisms of damage development during tensile loading, tensile specimens that were loaded to a predetermined increment of strain were sectioned parallel to the loading axis and the plane of random fibre orientation (Fig. 1) and mechanically polished using diamond paste down to $0.25 \mu \mathrm{m}$. Determination and quantification of the fraction of damaged fibres and of the distance separating two breaks along the same fibre were carried out in the composites at a magnification of $200 \times$ with a nominal resolution of the digitised pictures of $\pm 0.1 \mu \mathrm{m}$ using a Zeiss optical microscope equipped with a CCD camera. The fibre segments were selected manually, essentially using all fibres containing two or more breaks, visible in randomly selected micrographs. Fibres showing only one or zero breaks were thus not considered; these included essentially all fibres oriented at an angle to the plane of metallographic polish. The plane of polish is indicated in Fig. 1; it was chosen to coincide with the plane of fibre packing, so as to (i) contain the tensile axis and (ii) contain the greatest number of fibres lying within the plane of metallographic observation. Standard image analysis software (Optilab pro V 2.6, Graftek professional software, Austin, TX, USA) was then used to determine the length of fibre segments between two breaks, as well as their orientation. Measurements were carried out on at least 25 randomly selected metallographic areas; approximately 300 intact fibre segments situated between two breaks were thus measured for length and orientation in each composite at a given value of strain.

\subsection{Damage monitoring}

\subsubsection{Young's modulus-based damage parameter}

The measured reduction in Young's modulus during the tests was used to calculate a damage parameter, $D_{\mathrm{E}}$, conventionally defined as:

$D_{\mathrm{E}}=1-\frac{E(\varepsilon)}{E_{0}}$

with $E(\varepsilon)$ and $E_{0}$ being the Young's modulus at various total strains $\varepsilon$ and the initial Young's modulus, respectively [31]. The modulus at strain $\varepsilon, E(\varepsilon)$, was measured at strain intervals of $\Delta \varepsilon=0.5 \%$ overall strain by performing 12 unload-reload cycles between 30 and $70 \%$ of the maximum load reached before starting the cycling. The moduli determined from the last four unload-reload cycles at each increment were averaged to give the Young's modulus at the respective strain. This procedure, similar to that used in Refs. [30,32] was adopted since the first few unload-reload cycles showed traces of microplasticity that disappeared gradually with further cycling. Young's modulus was thus measured at typically 24 strain increments. Load-unload cycles were conducted at a crosshead speed of $0.3 \mathrm{~mm} / \mathrm{min}$. 


\subsubsection{Density-based damage parameter}

In a similar way, in order to evaluate the formation and evolution of pores within the composite, a second damage parameter is conventionally defined in terms of the composite density as:

$D_{\rho}=1-\frac{\rho(\varepsilon)}{\rho_{0}}$

where $\rho_{0}$ is the initial density of the composite prior to straining and $\rho(\varepsilon)$ is the density after applying an increment of strain [31]. To measure $D_{\rho}$, the specimen density was periodically measured during the tensile test after strain increments of either 0.5 or $1.0 \%$ using an Archimedean technique. This consisted in weighing the specimen with respect to a "dummy" sample of the same material, both in air and in an immersion fluid, before and after plastic straining [33]. A Sartorious MC 210P microbalance with a sensitivity of $\pm 10 \mu \mathrm{g}$ was used, with distilled water as the immersion fluid. Using this method, changes in specimen density of $\approx 0.005 \%$ could be measured reliably. Then, the damage parameter $D_{\rho}$ was back-calculated to the gauge length by making a correction for the unstrained (and hence constant) volume in the specimen shoulder. Density measurements on an isolated gripping section of a failed tensile specimen confirmed that there had been no density change and hence that the correction was justified.

\section{Results}

\subsection{General composite properties}

A typical microstructure of the as-infiltrated composites is given in Fig. 2. As seen, the fibre preform is well infiltrated, the as-produced composite being essentially void-free. The polished cross-section is in the plane of random orientation of the fibres, containing also the direction of tensile deformation during testing; as seen, fibres are indeed randomly oriented.

Three typical tensile curves for the composites are given in Fig. 3 (unload-reload cycles have been removed for clarity) showing reproducible stress-strain behaviour. The composites yield gradually and display good ductility, deforming to more than $10 \%$ total strain, with a flow stress slowly increasing from roughly 80 to $100 \mathrm{MPa}$. In some (but not all) of the composite samples, a region of concentrated plastic deformation, i.e., diffuse necking, was found to appear after $6-8 \%$ strain along the gauge length of the samples; this was ascertained by measuring cross-sectional dimensions of the samples at several locations along the gauge length after the tests.

\subsection{Indirect measures of damage}

Measured changes in the composite elastic modulus, $E$, are plotted versus strain $\varepsilon$ in Fig. 4 for three separate tensile samples. Similar trends are observed for all three samples: first, a
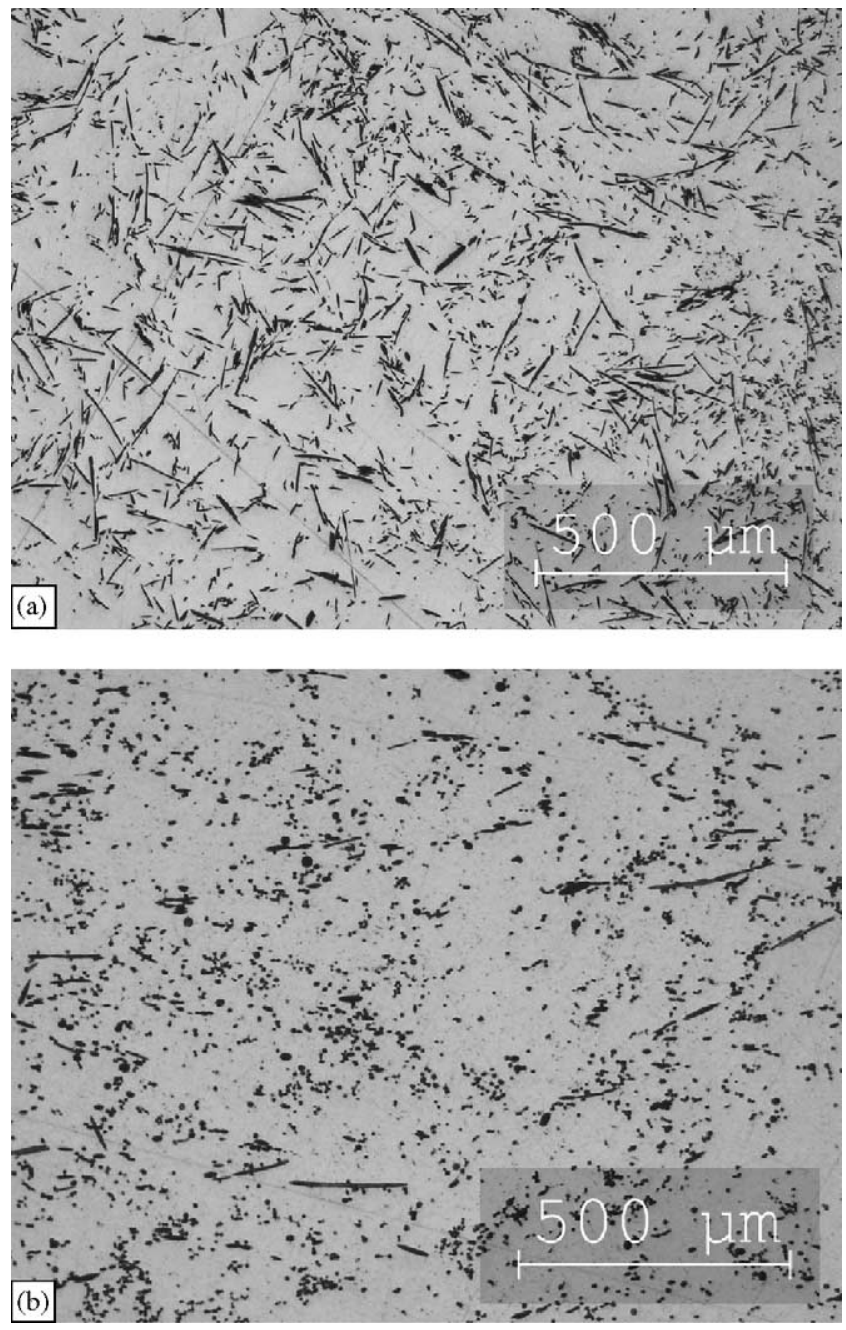

Fig. 2. Micrograph of the as-infiltrated composite in the plane of random fibre orientation (a) and perpendicular to it (b).

rapid decrease in elastic modulus until about $3 \%$ strain, followed by a second region where the curve levels off. Further straining past the transition between these two regions up to about $11 \%$ causes the elastic modulus to fall more gradually, by a further $3-5 \%$ of its initial value. For comparison, the Young's modulus evolution in the unreinforced matrix is given as well. The indicated data points are the average values of 12 different samples.

Density-based damage curves of $D_{\rho}$ versus strain $\varepsilon$ were measured on two specimens (Fig. 5). In one sample (Specimen 1), diffuse necking was observed to appear at strains above $6.5 \%$. Density data collected beyond this strain were, therefore, discarded. Instead, the gauge section was cut into three pieces of relatively uniform strain (the neck and its two neighbouring regions). The final density of each of these three regions was then measured, while its average strain was estimated by measurement of its cross-section. This yielded the three filled points in Fig. 5. Specimen 2, on the other hand, showed no necking during the test; hence, all data for this 


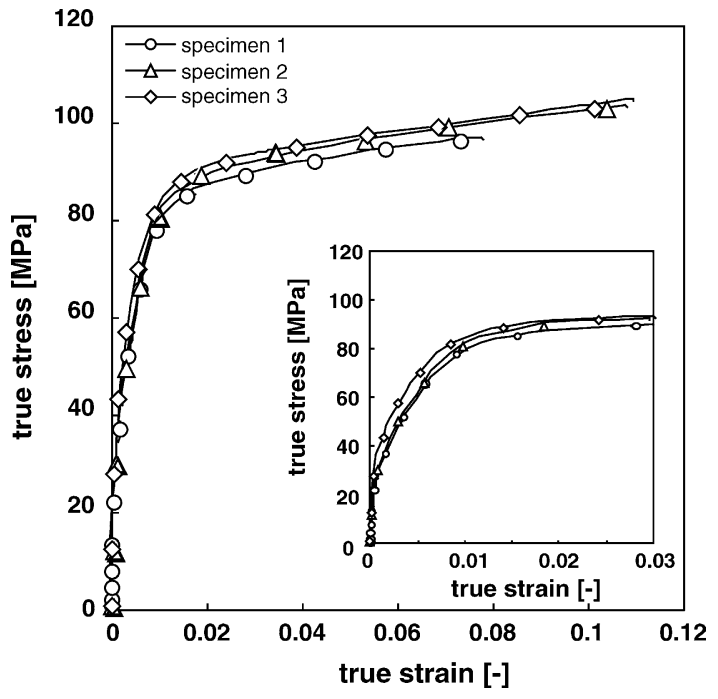

Fig. 3. Stress-strain behaviour of three different samples tested in tension exhibiting good reproducibility. The repeated unload-reload cycles for determination of Young's modulus at various strains have been eliminated from the graph for the sake of convenience. Note the typical bilinear behaviour at the beginning of the stress-strain curve (cf. inset).

specimen are plotted. As seen, the two sets of data superimpose, showing that the measurement method is reliable.

It can thus be concluded that a significant amount of void formation occurs only after a certain plastic strain (i.e., critical strain $\approx 3 \%$ ) has been imposed. It is also evident that void formation does not per se cause tensile instability, since a significant void content is present over a wide range of plastic strain before necking is observed [34].

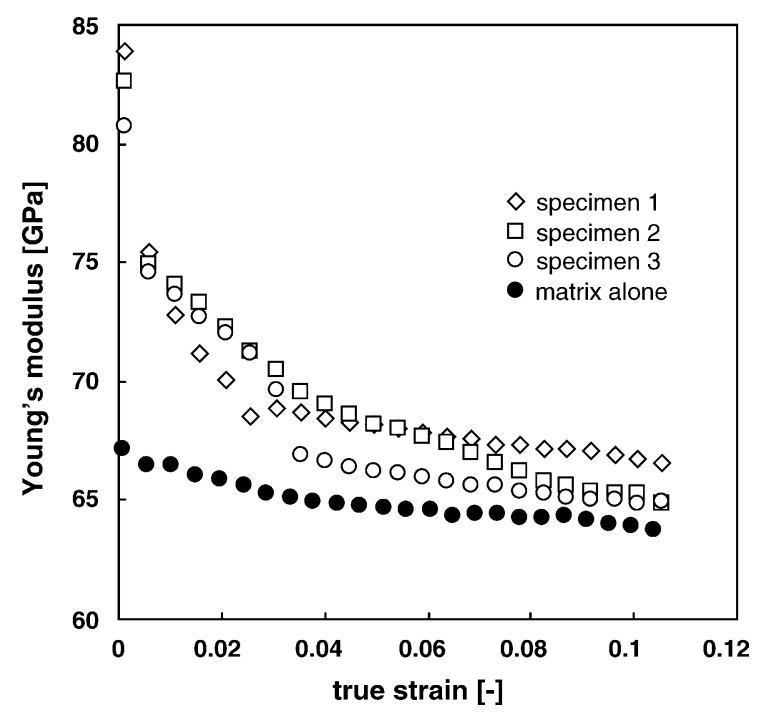

Fig. 4. Evolution of Young's modulus determined with repeated unloading-reloading cycles at various stages of a tensile test for three different composite specimens. A rapid drop in Young's modulus at low strains $(<3 \%)$ is followed by a regime of slower decrease at larger strains. The Young's modulus evolution averaged over 12 specimens of unreinforced matrix material is shown for comparison.

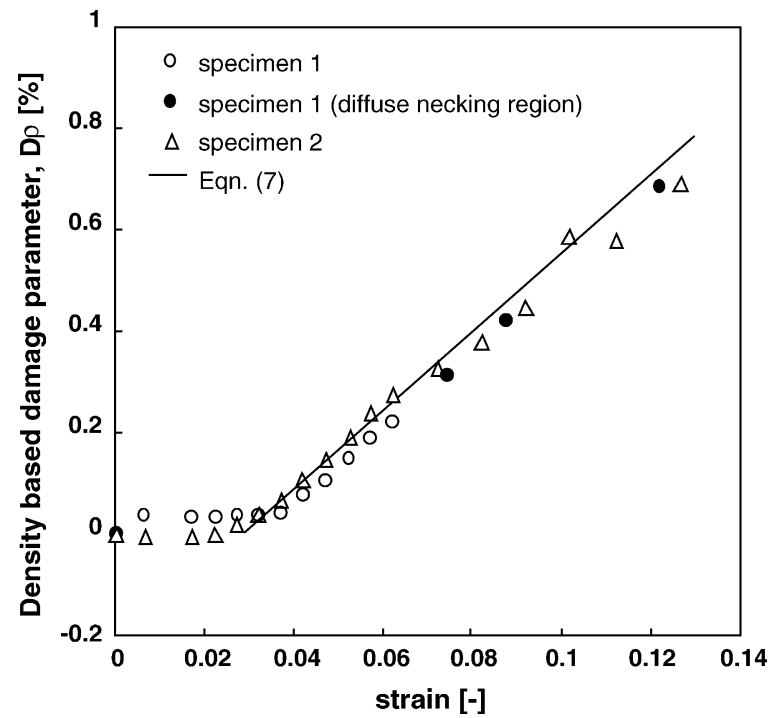

Fig. 5. Evolution of the density-based damage parameter with strain. After a first region with virtually unchanged density, the damage parameter increases linearly with strain.

\subsection{Direct measures of damage}

Metallographic observation reveals that damage takes two forms: (i) discrete transverse fibre breaks and (ii) voiding between the broken fibre segment ends (Fig. 6a). The number of breaks and the volume fraction voids opened between broken fibre ends can both be seen to increase with strain.

To quantify fibre breakage, the length of intact fibre segments between two fibre breaks were measured on polished cross-sections. These cross-sections were taken in the plane of random orientation of the fibres (thus containing the tensile axis) (Fig. 1); hence, several such fibre segments were visible and all fibre orientations were seen (Figs. 2 and 6).

These measurements are displayed in Fig. 7 for samples elongated to $0.1,0.4,1.6,3.0$ and $4.0 \%$ total strain. The orientation of the segments with respect to the tensile axis was divided into three classes: segments within an angle of $0-30^{\circ}$ of the tensile axis (Fig. 7a), those forming an angle between 30 and $60^{\circ}$ (Fig. 7b) and those making an angle above $60^{\circ}$ with the tensile axis (Fig. 7c). It can be seen that with increasing strain the average segment length decreases in all three orientation classes.

Comparison of these curves with the segment length distribution measured similarly on a sample taken to failure (at $13 \%$ strain) indicates that the segment length distribution for the class with misorientation $<30^{\circ}$ does not change after further deformation beyond the range for which data are reported in Fig. 7, i.e., the fragmentation of the fibres oriented within $30^{\circ}$ of the tensile axis is fully accomplished at around $3 \%$ of strain and does not evolve thereafter. For the two other orientation classes, i.e., for fibres inclined more than $30^{\circ}$ from the stress axis, the fibre fragmentation length distribution continues to evolve up to tensile fracture of the composite. 

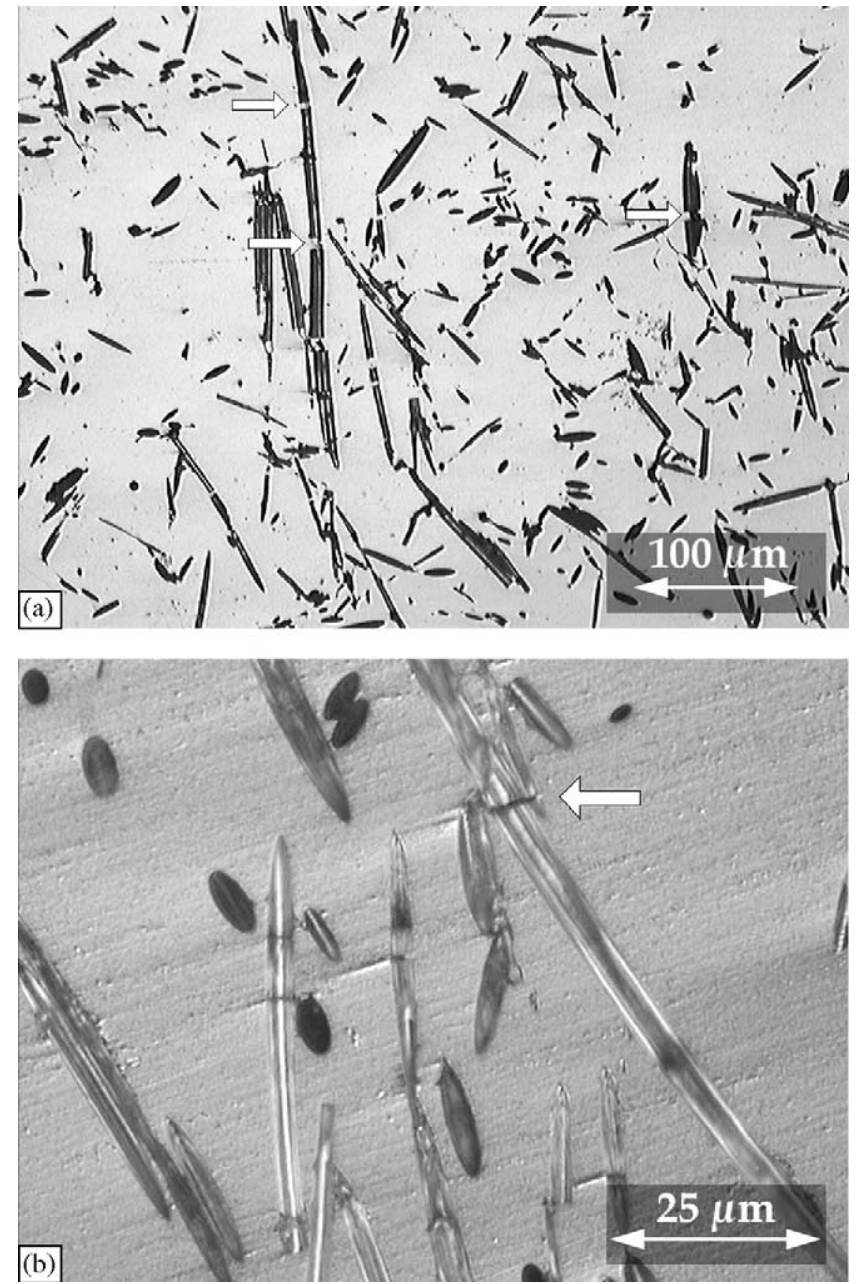

Fig. 6. (a) Optical micrograph of the composite after $8 \%$ strain showing the typical form of microstructural damage encountered in these composites: broken fibres and opening up of voids between the fibre ends at higher strain. (b) Fibre-fibre interactions leading to an increased number of fibre breaks. The tensile axis is vertical to the page.

\section{Discussion}

The tensile behaviour found here is consistent with that reported in an earlier study of the same material [35]. Taken together, observations and data of the present investigation lead to the following understanding of the evolution of internal damage during tensile deformation of Saffil reinforced aluminium.

Immediately after initial elastic deformation with the expected Young's modulus near $82 \mathrm{GPa}[3,25,35]$, there is, between 0.1 and $0.4 \%$ tensile strain, an initial stage of rapid stiffness reduction during which the Young's modulus decreases from 82 to $75 \mathrm{GPa}$, such that $D_{\mathrm{E}}$ increases rapidly to a value of 0.1 (Fig. 4). Interestingly, during this stage, there is no significant fibre fragmentation: all average segment length curves practically superimpose between 0.1 and $0.4 \%$ tensile strain (Fig. 5). Given the nominal fracture strain of $0.7 \%$ of the Saffil fibres, it is indeed not surprising that extensive fracture of the fibres has not begun at this early stage of deformation.
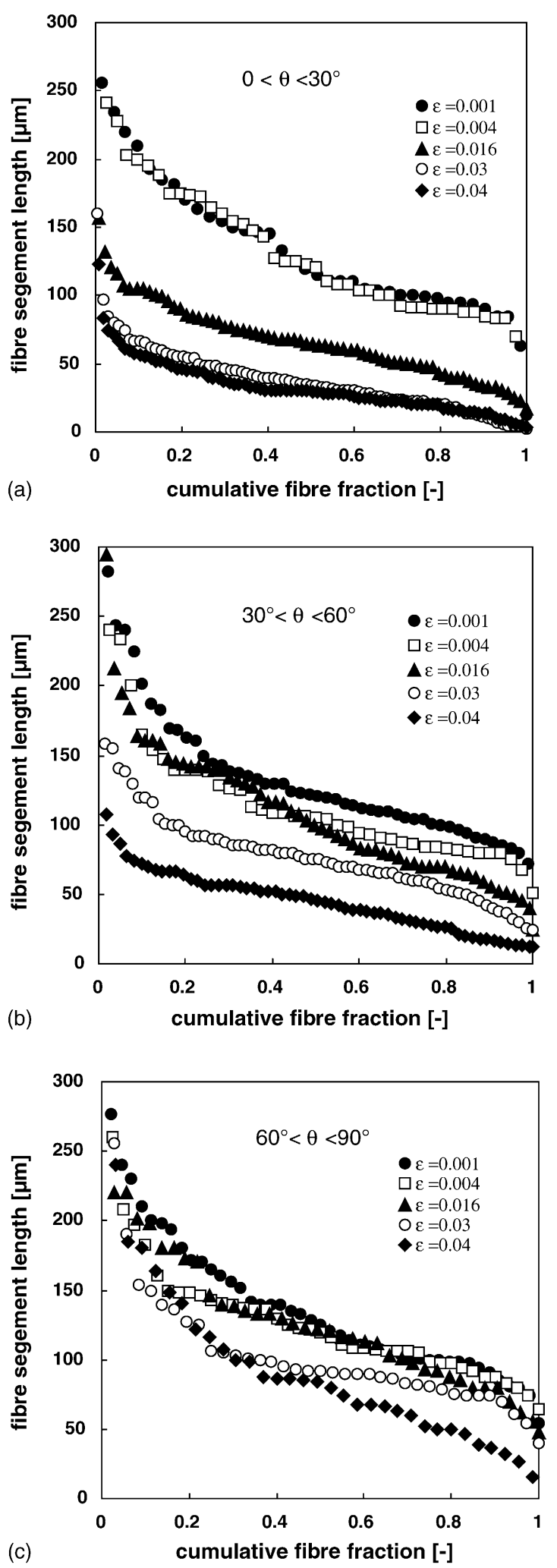

Fig. 7. Evolution of the average fibre fragment length as observed in the plane of planar random orientation for three different classes of fibre segment orientation with regard to the tensile axis: (a) $0-30^{\circ}$, (b) $30-60^{\circ}$ and (c) $>60^{\circ}$. 
The sharp initial drop in Young's modulus observed in this strain range must, hence, be due to some other effect, perhaps the breaking up of the binder bridges linking the fibres. Alternatively, given the relatively small stress range over which $E_{0}$ is measured, effects of bending and misalignment of the system could have contributed to an artificially high value of $E_{0}$. Indeed, in the measurement on the matrix alone a similar, yet much weaker, effect is seen, indicating that some but by far not all of the initial drop can be attributed to the varying stress range over which Young's modulus is measured.

A second stage extends between around 0.4 and $3 \%$ tensile strain. Here, the Young's modulus of the composites continues to drop (i.e., $D_{\mathrm{E}}$ continues to increase). The drop in Young's modulus in the composite in this range of strain is much stronger than in the matrix alone (Fig. 4), indicating that microstructural damage is accumulating in the composite. On the other hand, the density remains nearly unchanged ( $D_{\rho}$ remains near zero). There is thus a clear difference between the evolution of the two standard damage parameters, $D_{\mathrm{E}}$ and $D_{\rho}$. This was also observed in aluminium reinforced with densely packed alumina particles [32]. Metallographic evidence shows that, during this second stage, damage takes the form of progressive fibre breakage. Fragmentation in the well-aligned $\left(0-30^{\circ}\right)$ class of fibres proceeds rapidly and then saturates, as indicated in Fig. 7a by the unchanged segment length distribution for strains of 3 and $4 \%$ and, as noted above, for higher strain up to composite fracture. In fibres aligned between 30 and $60^{\circ}$ to the stress axis (Fig. 7b), there is also some damage by fibre fracture in this stage; however, the rate of fracture is well below that for fibres closer to the stress axis. For fibres nearly perpendicular to the tensile axis (angle in the range $60-90^{\circ}$ ), there is no significant damage by fracture (Fig. 7c). Overall, these observations agree with those of Clegg et al. [3], except for the fact that, in their work, fibre fracture was most pronounced between 2.5 and $3.5 \%$ strain. This difference can be rationalised by the fact that they did not distinguish between the fibre orientations (cf. Fig. 11 of ref. [3]). As can be seen in Fig. 12 of ref. [3], their findings on the fragmentation of fibres as a function of fibre orientation agree qualitatively with the results reported here, differences being probably due to differences in volume fraction and matrix alloy composition.

Two different modes of fibre fracture are observed in metallographic examination, namely (i) isolated fibre breaks attributable to load transfer from the matrix, found to occur first in fibres aligned along the loading axis and (ii) fracture due to fibre-fibre interaction (Fig. 6b) found in fibres adjacent to other fibres, often in fibre clusters and on some micrographs clearly attributable to bending of one fibre around its neighbours.

This second stage of composite deformation is thus dominated by the gradual fracture of fibres aligned close to the tensile axis; this is consistent with quantitative damage measures, as fibre fracture is expected to lower significantly the composite modulus while leaving its density essentially unaffected.
That most of the fibre fractures occur between composite strains of 0.4 and $1.6 \%$ strain (Fig. 7a) makes sense, given that the fibre fracture strain of Saffil ${ }^{\mathrm{TM}}$ fibres is around $0.7 \%$. This also agrees with the observation reported in a previous study of this composite that the slope of the composite stress-strain curves shows a rapid reduction near $1 \%$ strain $[5,23,35]$ as shown in the inset in Fig. 3; clearly a significant fraction of fibres break when the stress in their central section reaches their fracture strain, as predicted by standard composite shear-lag theory [36].

The critical fibre length, $l_{\mathrm{c}}$, is given by:

$l_{\mathrm{c}}=\frac{\sigma_{\mathrm{rupt}} r_{\mathrm{f}}}{\tau_{\mathrm{m}}}$

$l_{\mathrm{c}}$ is the shortest length of fibre of radius $r_{\mathrm{f}}$ that can be loaded to its fracture stress $\sigma_{\text {rupt }}$ by a plastic matrix having a flow stress in shear of $\tau_{\mathrm{m}}$ (cf. p. 252 in [37]). For the present composites, typical values of the relevant parameters are $\tau_{\mathrm{m}}=30 \mathrm{MPa}$ (the higher strain value of the stress-strain curve measured for the unreinforced Al matrix in Ref. [35]), $\sigma_{\text {rupt }}=2000 \mathrm{MPa}, r_{\mathrm{f}}=1.5 \mu \mathrm{m}[38,39]$. This yields $l_{\mathrm{c}}=100 \mu \mathrm{m}$. The average observed fibre segment length between breaks should theoretically be $0.75 l_{\mathrm{c}}$ [36], i.e., $75 \mu \mathrm{m}$. The observed average fibre segment length decreases from around 140 to $40 \mu \mathrm{m}$ as strain increases from 0.4 to $3 \%$ strain (Fig. 7a). This is of the right order of magnitude; however, the final average fibre segment length reached at the end of the fibre fragmentation process is nearer half the predicted value of $75 \mu \mathrm{m}$ (Fig. 7a). Several explanations exist: (i) additional breaks due to fibre-to-fibre interaction, as suggested by metallography (Fig. 6b) or (ii) the presence of sites of fibre strength lower than $2 \mathrm{GPa}$ [38]. The observed spread in fibre segment lengths and the range of composite strains over which fibre fracture progresses are obvious consequences of the large spread in fibre radius, fibre orientation and fibre strength (Figs. 2 and 6); another obvious cause is inhomogeneity in stress transfer to the fibres, particularly near fibre clusters.

Comparing the fibre fragmentation data and the measured evolution of Young's modulus, there is a clear coincidence of the strain $\varepsilon_{\mathrm{c}}$ at which the Young's modulus versus strain curve levels off and the strain at which the fragmentation of well aligned fibres is saturated, reinforcing the link made between the drop in Young's modulus and the fragmentation of fibres that are well aligned with regard to the tensile axis. While this is not surprising as such, it has the interesting corollary that, once such fibre fragmentation is accomplished, the rate of damage accumulation as quantified by $D_{\mathrm{E}}$ decreases sharply. As shown earlier [35], this can explain the comparatively high strain to failure observed in the present composite.

The marked difference between the Young's modulusbased and density-based damage parameters $D_{\mathrm{E}}$ and $D_{\rho}$-not only in their order of magnitude but also in their general evolution-resembles what was previously observed in particulate reinforced metal matrix composites with a high volume fraction of reinforcement [30]. As suggested by Fig. 6 , 

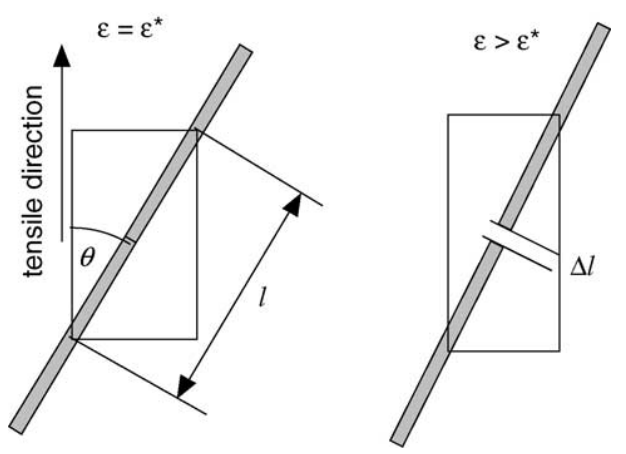

Fig. 8. Schematic model to determine the volume generated by separating the faces of the two fibre segments at a fibre break with the fibres accompanying the composite as it deforms.

this difference is basically due to the fact that the breaking of a fibre has an immediate effect on Young's modulus while its effect on density is only visible when voids have opened up across fibre ends at a break. A simple quantitative relation between the two damage measures can be estimated, somewhat similarly to the derivation in [30], as follows.

Consider a representative length $l$ of a fibre inclined at angle $\theta$ with respect to the tensile axis (Fig. 8). At a composite strain $\varepsilon^{*}$, the fibre breaks. Assume that the material around this length $l$ of fibre, thereafter, follows the overall composite upon further straining to strain $\varepsilon>\varepsilon^{*}, \varepsilon$ being large as compared to elastic strains in the fibre segments. The two ends of this fibre length then move one in relation to the other by a distance $\left[l\left(\varepsilon-\varepsilon^{*}\right) \cos \theta\right]$ parallel to the stress axis, and $\left[-l\left(\varepsilon-\varepsilon^{*}\right) \sin \theta / 2\right]$ perpendicularly to the stress axis. The fibre ends at the fibre break thus separate according to

$\frac{\Delta l}{l}=\left(\varepsilon-\varepsilon^{*}\right)\left(\frac{3}{2} \cos ^{2} \theta-\frac{1}{2}\right)$

using the approximation $(1+2 x)^{1 / 2} \approx 1+x$ for $x \ll 1$. The volume, $\Delta V$, generated in the form of a void across the fibre break is then

$\Delta V=A \Delta l=A l\left(\varepsilon-\varepsilon^{*}\right)\left(\frac{3}{2} \cos ^{2} \theta-\frac{1}{2}\right)$

where $A$ is the cross-section of the fibre. Integrating over all angles of orientation, one finds for the volume opened across separated fibre breaks

$\Delta V=A l\left(\varepsilon-\varepsilon^{*}\right) \frac{\pi}{4}$

and for the density-based damage parameter $D_{\rho}$ this leads to

$D_{\rho} \approx V_{\mathrm{f}}\left(\varepsilon-\varepsilon^{*}\right) \frac{\pi}{4}$

With the approximation that the fibre fragmentation process is finished after $3 \%$ strain, i.e., $\varepsilon^{*}=3 \%$, we expect a straight line for the evolution of $D_{\rho}$ with strain, the slope of which is $\left(\pi V_{\mathrm{f}} / 4\right)$. This linear relation is also indicated in Fig. 5 and is in good agreement with data, supporting the present interpretation of the evolution of $D_{\rho}$.

\section{Conclusions}

Damage accumulation in aluminium reinforced with 10 vol.\% Saffil alumina short fibre proceeds in three stages:

(i) Immediately after yield and up to a strain of $0.4 \%$, the Young's modulus of the composite drops by roughly $10 \%$. No visible fibre fragmentation occurs in this initial stage; it is proposed that the reduction in modulus is mainly caused by break-up of the binder bridging the fibres.

(ii) From 0.4 to around 3\% strain, the Young's modulus decreases roughly by another $10 \%$, while the density remains constant. In this range of deformation, the Young's modulus drop in the composite is much stronger than in the reference matrix material. Microstructural damage in this range of deformation progresses mainly by fibre fragmentation, which is completed in fibres aligned close to the tensile axis when the strain reaches $3 \%$. The larger the misorientation between fibres and the tensile axis, the higher is the strain required to complete the fibre fragmentation process. Comparison of the Young's modulus evolution and the advancement of the fragmentation process with strain suggests that the drop in Young's modulus is mostly due to the fragmentation of short fibres roughly aligned with the tensile axis.

(iii) Beyond 3\% strain up to the composite fracture strain of $10-12 \%$, the rate of decrease of Young's modulus with strain is far lower and close to that observed in the unreinforced matrix. The density-based damage parameter $D_{\rho}$ increases roughly linearly with strain. A simple geometrical model based on void formation between separating faces at fibre breaks accounts for the evolution of $D_{\rho}$ in this range of composite deformation.

\section{Acknowledgements}

One of the authors (R. Tavangar) gratefully acknowledges sponsoring of his research by the Ministry of Higher Education of the Islamic Republic of Iran during his stay at the Swiss Federal Institute of Technology. This research was also funded by core funding of the Swiss Federal Institute of Technology in Lausanne.

\section{References}

[1] D.A. Gerard, T. Suganuma, P.H. Mikkola, A. Mortensen, in: R. Abbaschian, H. Brody, A. Mortensen (Eds.), Merton C. Flemings Symposium on Solidification and Materials Processing, TMS, Warrendale, PA, 2001, pp. 475-488.

[2] A. Evans, C. SanMarchi, A. Mortensen, Metal Matrix Composites in Industry-An Introduction and a Survey, Kluwer Academic Publishers, Dordrecht, NL, 2003.

[3] W.J. Clegg, I. Horsfall, J.F. Mason, L. Edwards, Acta Metall. 36 (1988) 2151-2159. 
[4] I.W. Hall, in: N. Hansen, D. Juul Jensen, T. Leffers, H. Lilholt, T. Lorentzen, A.S. Pedersen, O.B. Pederson, B. Ralph (Eds.), Metal Matrix Composites-Processing, Microstructure and Properties, 12th Ris $\emptyset$ International Symposium on Materials Science, Ris $\emptyset$ National Laboratory Roskilde, Danemark, 1991, pp. 367-372.

[5] W.J. Baxter, A.K. Sachdev, Metall. Mater. Trans. 30A (1999) $815-823$.

[6] N.J. Musson, T.M. Yue, Mater. Sci. Eng. A135 (1991) 237-242.

[7] S. Bengtsson, R. Warren, Mater. Sci. Technol. 9 (1993) 319327.

[8] D.J. Towle, C.M. Friend, Mater. Sci. Technol. 9 (1993) 35-41.

[9] C.M. Friend, J. Mater. Sci. 22 (1987) 3005-3010.

[10] J.M. O’Rourke, R.S. Bushby, V.D. Scott, Compos. Sci. Technol. 56 (1996) 1071-1077.

[11] J.M. O’Rourke, R.S. Bushby, V.D. Scott, Compos. Sci. Technol. 56 (1996) 957-965.

[12] V.D. Scott, J.M. O’Rourke, R.S. Bushby, Composites B 30 (1999) $1-7$.

[13] S. Harris, T.-E. Wilks, in: A.R. Bunsell, P. Lamicq, A. Massiah (Eds.), First European Conference on Composite Materials, ECCM1, AEMC, Bordeaux, France, 1985, pp. 595-603.

[14] C.M. Friend, Scripta Metall. 23 (1989) 33-37.

[15] M. Pahutova, J. Brezina, K. Kucharova, V. Sklenicka, T.G. Langdon, Mater. Lett. 39 (1999) 179-183.

[16] A. Dlouhy, N. Merk, G. Eggeler, Acta Metall. Mater. 41 (1993) 3245-3256.

[17] A. Dlouhy, G. Eggeler, N. Merk, Acta Metall. Mater. 43 (1995) 535-550.

[18] A.F. Whitehouse, H.M.A. Winand, T.W. Clyne, Mater. Sci. Eng. A242 (1998) 57-69.

[19] A. Yawny, G. Kausträter, B. Skrotzki, G. Eggeler, Scripta Mater. 46 (2002) 837-842.

[20] W. Shen, D. Chu, L.-H. Peng, J. Xu, Eng. Frac. Mech. 51 (1995) 479-486.

[21] H.-Z. Ding, H. Biermann, O. Hartmann, Int. J. Fatigue 25 (2003) 209-220.

[22] H.-Z. Ding, H. Biermann, O. Hartmann, Compos. Sci. Technol. 62 (2002) 2189-2199.
[23] C. Badini, P. Fino, M. Musso, P. Dinardo, Mater. Chem. Phys. 64 (2000) 247-255.

[24] T. Beck, K.-H. Lang, D. Löhe, Mater. Sci. Eng. A319-321 (2001) 662-666.

[25] G. Chadwick, in: J. Füller, G. Grüninger, K. Schulte, A.R. Bunsell, A. Massiah (Eds.), Fourth European Conference on Composite Materials, Elsevier Science Publishers, London, UK, 1990, pp. 3-13.

[26] K.-S. Sohn, K. Euh, S. Lee, I. Park, Metall. Mater. Trans. 29A (1998) 2543-2554.

[27] F.P. Kehoe, G.A. Chadwick, Mater. Sci. Eng. A135 (1991) 209-212.

[28] A.F. Whitehouse, T.W. Clyne, Acta Metall. Mater. 41 (1993) 1701-1711.

[29] M. Vedani, E. Gariboldi, Acta Mater. 44 (1996) 3077-3088.

[30] M. Kouzeli, L. Weber, C. SanMarchi, A. Mortensen, Acta Mater. 49 (2001) 497-505.

[31] J. Lemaitre, A Course on Damage Mechanics, second ed., Springer, Berlin, 1996.

[32] M. Kouzeli, L. Weber, C. SanMarchi, A. Mortensen, Acta Mater. 49 (2001) 3699-3709.

[33] R.T. Ratcliffe, Br. J. Appl. Phys. 16 (1965) 1193.

[34] L. Weber, M. Kouzeli, C. SanMarchi, A. Mortensen, Scripta Mater. 41 (1999) 549-551.

[35] R. Tavangar, S. Nategh, L. Weber, Mater. Sci. Technol. 20 (2004) 1645-1648.

[36] H. Fukuda, Y. Takao, in: A. Kelly, C. Zweben (Eds.), Comprehensive Composite Materials. Reinforcement Materials and General Theories, vol. 1, Elsevier, Oxford, UK, 2000, pp. 377-401.

[37] A. Kelly, N.H. Macmillan, Strong Solids, third ed., Oxford University Press, Oxford, UK, 1986

[38] J. Dinwoodie, I. Horsfall, in: F.L. Matthews, N.C.R. Buskell, J.M. Hodgkinson, J. Morton (Eds.), Joint Sixth International Conference on Composite Materials and Second European Conference on Composite Materials, Elsevier Applied Science, Barking, UK, 1987, pp. 2.390-2.401

[39] J. Dinwoodie, E. Moore, C.A.J. Langman, W.R. Symes, in: W.C. Harrigan Jr., J. Strife, A.K. Dhingra (Eds.), Fifth International Conference on Composite Materials, TMS, Warrendale, PA, 1985, pp $671-685$. 